Check for updates

Cite this: RSC Adv., 2019, 9, 31062

\title{
Surface modification of an aramid fiber via grafting epichlorohydrin assisted by supercritical $\mathrm{CO}_{2}$
}

\begin{abstract}
Xiaoma Ding, ${ }^{a}$ Haijuan Kong, ${ }^{b}$ Mengmeng Qiao, ${ }^{a}$ Luwei Zhang (D) a and Muhuo Yu (D) *a
In order to improve the interface combination property between an aramid fiber (AF) and an epoxy resin matrix, the surface modification of AF with epichlorohydrin $(\mathrm{ECH})$ assisted by supercritical $\mathrm{CO}_{2}\left(\mathrm{ScCO}_{2}\right)$ was investigated. The fiber surface was characterized by X-ray photoelectron spectroscopy (XPS), scanning electron microscopy (SEM) and dynamic contact angle (DCA) analysis. At the same time, we utilized interfacial shear strength (IFSS) and interlaminar shear strength (ILSS) to characterize the bond strength between the fiber and epoxy resin. An ideal modification effect of the fiber surface was acquired when the fiber treated with $\mathrm{ECH}$ in $\mathrm{ScCO}_{2}$ compared with the fiber treated in pure $\mathrm{ScCO}_{2}$. The results showed that $\mathrm{ECH}$ could be successfully grafted onto the fiber surface under an anhydrous aluminum chloride $\left(\mathrm{AlCl}_{3}\right)$ catalyst in $\mathrm{ScCO}_{2}$, and the relative content of oxygen on the fiber surface increased after modification; simultaneously, the morphology of the fiber surface became rougher and the fiber's wettability was upgraded. Finally, the IFSS property of the fiber with the epoxy resin increased, and the ILSS property of the AF-reinforced resin composites was also improved compared with those of the untreated materials.
\end{abstract}

Received 15th July 2019

Accepted 14th September 2019

DOI: 10.1039/c9ra05395f

rsc.li/rsc-advances of the composite materials. ${ }^{6}$ In other words, the destruction of the AF-reinforced composite products during the service process is mainly attributed to the weak combination between the fiber and the matrix. ${ }^{7}$

In response to the above drawbacks, many researchers have been trying different methods to modify the AF surface including surface coating, plasma treatment, and chemical grafting, which mainly introduce more polar components or increase the roughness of the fiber surface, achieving satisfactory results. ${ }^{8-12}$ Among these modification methods, the plasma treatment has a good modification effect in improving the bond properties between $\mathrm{AF}$ and the resin by etching the fiber surface, but the reduction of the fiber strength is unavoidable. ${ }^{12}$ In addition, compared with the modification effect of the surface coating method, the chemical grafting method can result in a chemical bond with the fiber while increasing the functional groups on the fiber surface. This is conducive to the combination between the fiber and resin and obtaining an excellent interface, thereby prolonging the service life of the composite materials. However, the grafting effect of the traditional chemical modification method is not so ideal to modify large quantities of fiber bundles and fabrics due to the poor penetration of the modifier. Therefore, in order to effectively modify the high-volume fiber products and maximally retain the fiber strength, some other modification methods must be considered.

Supercritical $\mathrm{CO}_{2}\left(\mathrm{ScCO}_{2}\right)$ having the critical temperature and pressure of $31{ }^{\circ} \mathrm{C}$ and $7.4 \mathrm{MPa}$, respectively, ${ }^{\mathbf{1 3 , 1 4}}$ has been confirmed as an environmentally friendly medium for carrying small molecules to the surface or even to the interior of fibers
${ }^{a}$ State Key Laboratory for Modification of Chemical Fibers and Polymer Materials,
College of Materials Science and Engineering, Shanghai Key Laboratory of
Lightweight Composite, Donghua University, Shanghai 201620, China. E-mail:
dhuyumuhuo@163.com
${ }^{b}$ School of Materials Engineer, Shanghai University of Engineer Science, Shanghai 201620, China 
owing to its superior swelling, plasticization and convenient conversion capability between the liquid and gas. ${ }^{15-22}$ Kong et al. used $\mathrm{ScCO}_{2}$ to carry hexamethylene diisocyanate and toluene-2, 4diisocyanate to the amorphous region of AF to enhance the degree of crystallization of $\mathrm{AF}^{\mathbf{1 5 , 2 3}}$ Yoshida et al. proposed a new electroplating method of nickel in an emulsion of $\mathrm{ScCO}_{2}$ to enhance the uniformity of the plated film. ${ }^{24}$ In view of these, $\mathrm{ScCO}_{2}$ can be tentatively used as a reaction medium for the uniform modification of the fiber surface without reducing the fiber strength. Epichlorohydrin (ECH) is an important oxygen-containing organic synthesis raw material used primarily in the manufacture of epoxy resins and as a modifier. ECH contains a large number of oxygencontaining functional groups, which can be grafted onto the benzene ring to increase the activity and roughness of the fiber surface. Wu and co-workers showed that $27.88 \%$ improvement in the interlaminar shear strength (ILSS) property of F-12 AFreinforced E-51 epoxy resin composites could be achieved by ECH grafting using the traditional chemical method. Among them, the fibers were previously treated with a mixture of potassium hydroxide and ethanol to break the fiber molecule chains and then reacted with $\mathrm{ECH} .{ }^{25}$ Liu and co-workers showed that the ILSS property of the AF-reinforced epoxy matrix composites increased by $50 \%$ when the fibers were treated with $\mathrm{ECH}$ using Friedel-Crafts alkylation; ${ }^{26}$ it is a reaction in which a hydrogen atom on the aromatic ring is replaced by an alkyl group of the haloalkane in the presence of a Lewis acid such as anhydrous aluminum trichloride $\left(\mathrm{AlCl}_{3}\right)$. The related literature shows that $\mathrm{ScCO}_{2}$ can dissolve most organic compounds including heptachlor epoxide ${ }^{27,28}$ and the reaction activity in the $\mathrm{ScCO}_{2}$ atmosphere is more active under low temperatures than the chemical reaction at high temperatures due to the strong diffusion ability of $\mathrm{ScCO}_{2} \cdot{ }^{29}$ In order to improve the uniformity and continuity of the grafting effect, $\mathrm{ScCO}_{2}$ was selected as a modification medium.

In this article, we tentatively used $\mathrm{ECH}$ under the catalysis of $\mathrm{AlCl}_{3}$ in $\mathrm{ScCO}_{2}$ to modify the surface of the AF bundles and fabrics in the reactor, providing ideas for modifying large quantities of fiber products and thereby increasing the interlaminar properties of the composite materials in a broad field. In order to rule out the influences of pure $\mathrm{ScCO}_{2}$ on the modification effect of the fiber surface treated with $\mathrm{ECH}$ in $\mathrm{ScCO}_{2}$, we simultaneously carried out a parallel reaction of fibers treated in pure $\mathrm{ScCO}_{2}$.

\section{Materials and methods}

\subsection{Materials}

AF bundles made up of 1000 monofilaments with linear density of 3.56 dtex and diameter of $16.65 \mu \mathrm{m}$ as well as AF-weaved fabrics (Style Mt-9120, $200 \mathrm{~g} \mathrm{~m}^{-2}$ ) with ply of $0^{\circ}$ and $90^{\circ}$ were provided by the Zhonglan Chenguang Chemical Research Institute (Sichuan, China). $\mathrm{CO}_{2}$ gas with purity of $99.99 \%$ was purchased from Shanghai Jiali Gas Co., Ltd. (Shanghai, China). Acetone ( $\geq 99.50 \%)$, $\mathrm{AlCl}_{3}$ (analytical grade) and ECH with a boiling point of $117.9^{\circ} \mathrm{C}$ were all acquired from the Sinopharm Chemical Reagent Beijing Co., Ltd. (Beijing, China). A resin system containing the epoxy resin E-51 and the corresponding curing agent M-36 with a stoichiometric mass ratio of $100: 25$ and curing system of $120^{\circ} \mathrm{C}$ and $2 \mathrm{~h}$ was obtained from Shanghai Fangye Chemical Co., Ltd. (Shanghai, China).

\subsection{Modification process and experimental principle of AF surface}

A schematic diagram of the device of the AF surface treated with $\mathrm{ECH}$ in the $\mathrm{ScCO}_{2}$ reactor is shown in Fig. 1a. The entire process can be summarized as follows: first, the fibers were washed repeatedly with acetone through a circulating condensing device at $80^{\circ} \mathrm{C}$ for $12 \mathrm{~h}$, followed by drying in a vacuum oven at $80{ }^{\circ} \mathrm{C}$ until there were no changes in the fiber quality. Next, $2 \mathrm{~g}$ of the cleaned fiber bundles and ten layers of fabrics as well as $0.1 \mathrm{~g}$ of $\mathrm{AlCl}_{3}$ as the catalyst were tightly fixed onto every platform (Fig. 1b) connected to the stir bar of the reactor with a volume of $10 \mathrm{~L}$ customized by Tianjin Yantu Experimental Instrument Development Co., Ltd. (Tianjin, China). Then, about $2 \mathrm{MPa}$ of $\mathrm{CO}_{2}$ gas was transported from the $\mathrm{CO}_{2}$ cylinder to the reactor to remove the air by a booster pump with a pressurization ratio of 64 when the temperature of the reactor rose to $120{ }^{\circ} \mathrm{C}$ and exhausted the gas after a while; this operation was repeated twice. After the air in the reactor was completely discharged, $50 \mathrm{ml}$ of $\mathrm{ECH}$ was sent into the reactor with a syringe through the feed port on the reaction vessel; this was followed by closing the feed port, starting the stir bar to move at a speed of $300 \mathrm{rpm}$ to make $\mathrm{ScCO}_{2}$ flow in the reactor and beginning to pressurize the pressure to $10 \mathrm{MPa}$ when the temperature rose to $120{ }^{\circ} \mathrm{C}$ again. Finally, the reaction was carried out for $2 \mathrm{~h}$ by relying on $\mathrm{ScCO}_{2}$ to drive $\mathrm{ECH}$ to the fiber surface when the temperature and pressure in the reactor were maintained at $120^{\circ} \mathrm{C}$ and $10 \mathrm{MPa}$, respectively. After the reaction, the samples were washed with acetone at $60{ }^{\circ} \mathrm{C}$ for $40 \mathrm{~min}$ in an ultrasonic cleaning machine, followed by drying in a vacuum oven at $80^{\circ} \mathrm{C}$ for $2 \mathrm{~h}$; then, they were collected for various characterizations. Fig. 2 shows the diagram of the modification process of the $\mathrm{AF}$ surface with $\mathrm{ECH}$ in the $\mathrm{ScCO}_{2}$ medium. Fig. 3 shows the mechanism of the main reaction of $\mathrm{ECH}$ grafted onto the fiber surface under the catalysis of $\mathrm{AlCl}_{3}$, from which we inferred that $\mathrm{H}$ on the benzene ring connected to $\mathrm{N}-\mathrm{H}$ of the molecular chain was substituted by an epoxy alkyl functional group from $\mathrm{ECH}$, thereby providing an oxygen-containing functional group on the fiber surface.

\subsection{Preparation of micro-debonding test samples and AF- reinforced composites}

Several single AFs were randomly selected from the fiber bundles and glued to a rectangular metal frame with an adhesive. Then, a trace amount of the E-51 resin system was dropped onto the monofilament and cured in a vacuum oven at $120{ }^{\circ} \mathrm{C}$ for $2 \mathrm{~h}$. The AF-reinforced resin composites were prepared by the vacuum-assisted resin infusion molding technology ${ }^{30}$ with the same curing process as the resin system.

\subsection{Characterization}

The functional groups and elemental analysis of the fiber surface were examined by an X-ray photoelectron spectrometer (XPS, Escalab 250Xi, Thermo Scientific, USA) with the excitation 

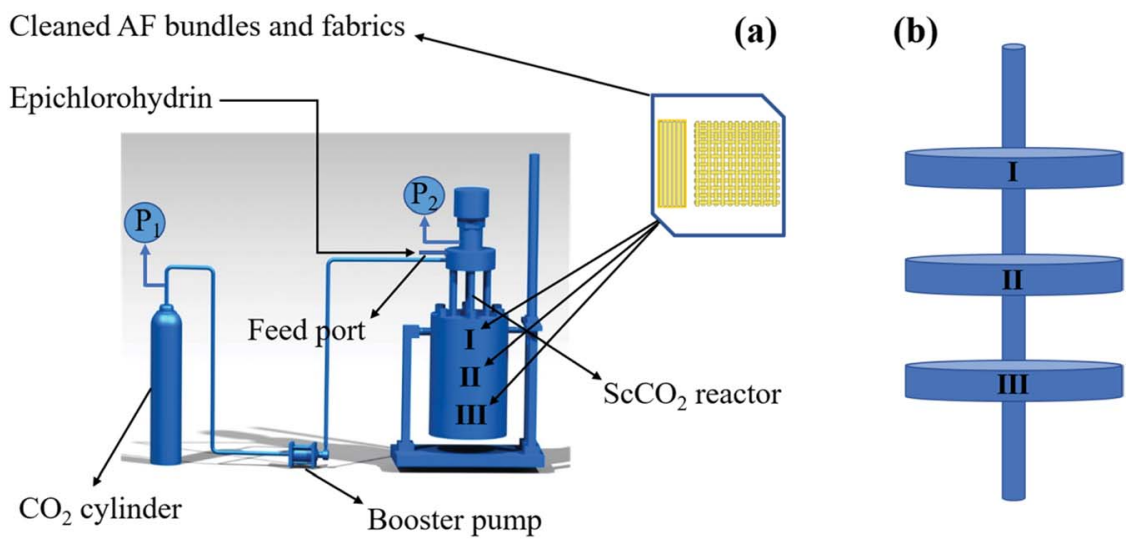

Fig. 1 Schematic diagram of the device containing AF surfaces treated with $\mathrm{ECH}$ in an $\mathrm{ScCO}_{2}$ reactor (a) and the platform to fix the fiber (b).

source and standard source of $\mathrm{Mg}$ and $\mathrm{K} \alpha$ rays, respectively, at a voltage of $8.0 \mathrm{kV}$ and power of $240 \mathrm{~W}$. The morphologies of the fiber surface and fracture cross-section of the composites were observed by scanning electron microscopy (SEM, Hitachi S4700 , Japan). All samples were pre-coated with a layer of gold and the voltage and current were adjusted to $12.5 \mathrm{kV}$ and $10 \mu \mathrm{A}$, respectively, during the test. The dynamic contact angle (DCA) and surface free energy of the fiber surface were measured with the test media of deionized water and diiodomethane by a CA meter (DCAT21, Data-Physics Instrument, Germany) at an ambient temperature. The DCA and surface free energy including the dispersive and polar components were calculated based on the Wilhelmy's equation ${ }^{31}$ and the Owens-Wendt model, ${ }^{32}$ respectively. The interfacial shear strength (IFSS) property of the fiber with the resin system was tested using a setup in which the epoxy system droplet was placed between two metal blades in advance, as shown in Fig. 4, and stretched to obtain the maximum load at a loading speed of 0.06 $\mathrm{mm} \mathrm{min}^{-1}$. The IFSS property of the fiber with the resin system was calculated using eqn (1): ${ }^{33}$

$$
\text { IFSS }=\frac{F}{\pi D L}
$$

here, $F$ is the maximum load during the test $(\mathrm{N}), D$ is the fiber's diameter $(\mathrm{mm})$, and $L$ is the embedded length $(\mu \mathrm{m})$.

The ILSS property of the AF-reinforced composites was tested through a universal test machine (5960, Instron, USA) by a three-point bending technology with a span length of $16 \mathrm{~mm}$ and cross-head speed of $10 \mathrm{~mm} \min ^{-1}$ according to ASTM D2344 standard. $^{34}$ The ILSS property of the AF-reinforced composites was calculated using eqn (2):

$$
\text { ILSS }=\frac{3 P}{4 b h}
$$

here, $P$ is the maximum load in the test (N), and $b$ and $h$ are the width $(\mathrm{mm})$ and thickness $(\mathrm{mm})$ of each sample before the test, respectively.

\section{Results and discussion}

\subsection{XPS analysis}

Fig. 5 reveals the XPS spectra of the untreated and modified AF surfaces, and the corresponding chemical composition of the fiber surface is presented in Table 1 . The peaks at the binding energies of 285,400 and $532 \mathrm{eV}$, corresponding to carbon, nitrogen and oxygen, represent the main elemental composition of the untreated AF surface..$^{35}$ There were no significant changes in the chemical composition of the fiber surface when the fibers were treated with pure $\mathrm{ScCO}_{2}$, as summarized in Table 1, indicating that pure $\mathrm{ScCO}_{2}$ had no obvious modification effects on the fiber surface. After treatment with ECH in $\mathrm{ScCO}_{2}$, a new binding energy peak appeared at $201 \mathrm{eV}$, corresponding to chlorine. This is because the Friedel-Crafts reaction is a reversible reaction, allowing some ECH species to remain on the fiber surface after the reaction. Furthermore, we observed increase in the

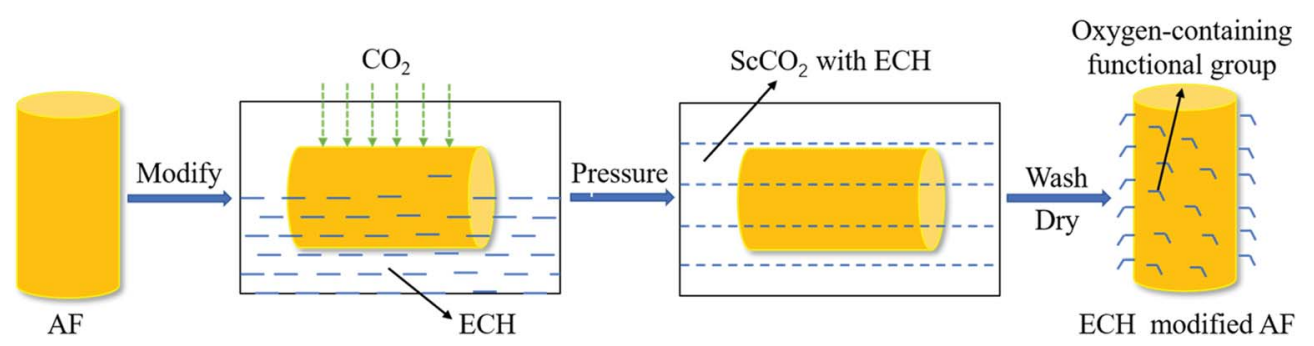

Fig. 2 Process diagram of the modification of the AF surface with $\mathrm{ECH}$ in $\mathrm{ScCO}_{2}$ medium. 
<smiles>ClCC1CO1</smiles><smiles>[R]N([14CH3])c1ccc(NC(=O)c2ccc(C(=O)C(C)(C)C)cc2)cc1</smiles><smiles>[R2]C(C)Nc1ccc(NC(=O)c2ccc(C(=O)C(C)(C)CC)cc2)c(C2(C)CO2)c1</smiles>

$\left(\mathrm{AlCl}_{4}\right)^{\ominus}+\mathrm{H}^{\oplus} \longrightarrow \mathrm{AlCl}_{3}+\mathrm{HCl}$

Fig. 3 Mechanism of the main reaction of $E C H$ grafted onto the $A F$ surface using the catalyst $\mathrm{AlCl}_{3}$.

relative concentrations of oxygen and the $\mathrm{O} / \mathrm{C}$ ratio from $12.68 \%$ and 0.164 to $22.47 \%$ and 0.317 , respectively, after the fiber was treated with $\mathrm{ECH}$ in $\mathrm{ScCO}_{2}$, thereby enhancing the activity of the fiber surface, which was beneficial for bonding with the resin system..$^{12,36-38}$

Furthermore, in order to observe the changes in the functional groups before and after modification, Fig. 6 presents the $\mathrm{C}_{1 \mathrm{~s}}$ peak spectra of the untreated and modified AF surfaces. The $\mathrm{C}_{1 \mathrm{~s}}$ peak spectrum of the untreated $\mathrm{AF}$ surface indicates the presence of $70.25 \% \mathrm{C}-\mathrm{C}, 15.74 \% \mathrm{C}-\mathrm{N}$ and $14.01 \% \mathrm{C}=\mathrm{O}$ at $284.8,285.9$ and $288.0 \mathrm{eV}$, respectively, which are the main functional groups on the AF surface, as shown in Fig. 6a. There were no significant changes in the functional groups of the fiber surface treated without $\mathrm{ECH}$ in $\mathrm{ScCO}_{2}$, as depicted in Fig. 6b. However, as we can see from Fig. 6c, the new functional group $\mathrm{C}-\mathrm{O}$ with the content of $13.75 \%$ and a peak at $286.3 \mathrm{eV}$ implied that $\mathrm{ECH}$ was successfully grafted onto the fiber surface assisted

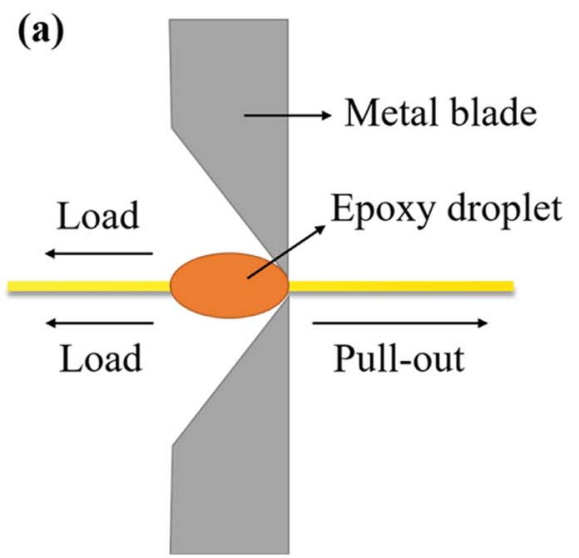

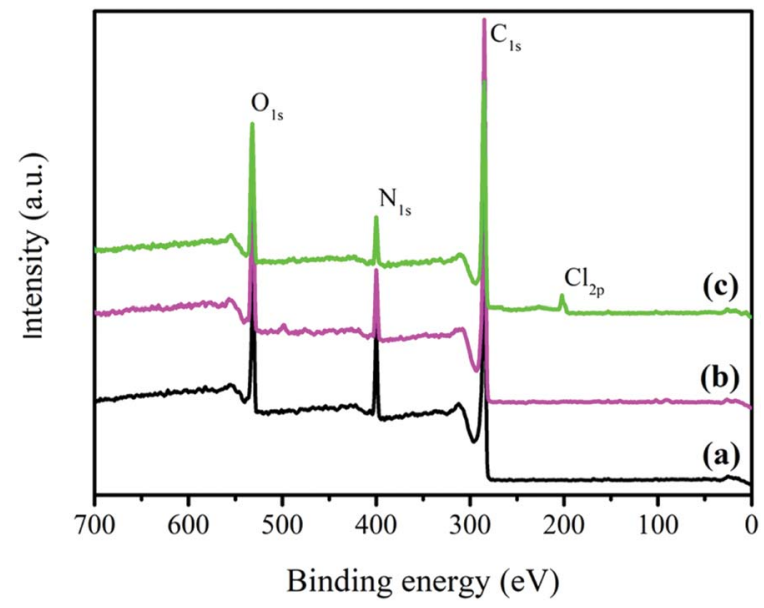

Fig. 5 XPS spectra of the related AF surfaces: (a) the untreated AFs, (b) AFs treated in pure $\mathrm{ScCO}_{2}$ and (c) AFs modified with $\mathrm{ECH}$ in $\mathrm{SCCO}_{2}$.

Table 1 Elemental analysis of the AF surface at different modification conditions

\begin{tabular}{|c|c|c|c|c|c|c|}
\hline \multirow[b]{2}{*}{ Samples } & \multicolumn{4}{|c|}{$\begin{array}{l}\text { Chemical composition } \\
\text { (at. mol\%) }\end{array}$} & \multicolumn{2}{|c|}{ Atomic ratio } \\
\hline & $\mathrm{C}$ & $\mathrm{O}$ & $\mathrm{N}$ & $\mathrm{Cl}$ & $\mathrm{N} / \mathrm{C}$ & $\mathrm{O} / \mathrm{C}$ \\
\hline Untreated AFs & 77.49 & 12.68 & 9.79 & 0.04 & 0.126 & 0.164 \\
\hline $\mathrm{AFs}$ in $\mathrm{ScCO}_{2}$ & 76.54 & 13.52 & 9.92 & 0.02 & 0.130 & 0.177 \\
\hline AF-ECP in $\mathrm{ScCO}_{2}$ & 70.84 & 22.47 & 6.33 & 0.36 & 0.089 & 0.317 \\
\hline
\end{tabular}

by $\mathrm{ScCO}_{2}$. These changes correspond to the improvement in the $\mathrm{O} / \mathrm{C}$ ratio given in Table 1.

\subsection{SEM analysis}

The surface morphology of AF before and after modification was examined by SEM, as depicted in Fig. 7. The untreated AF surface was rather smooth, as shown in Fig. 7a, obviously leading to poor interfacial adhesion with the resin system. It is

Fig. 4 . Schematic diagram of the pull-out test setup of single AF (a) and microsphere coating size and fiber diameter (b).

(b)

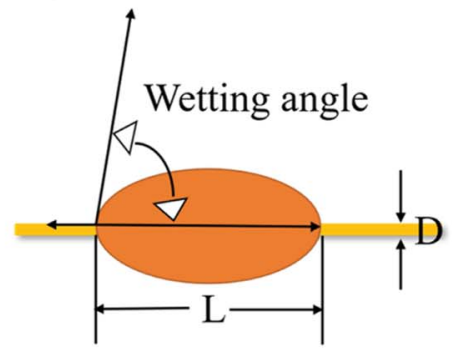



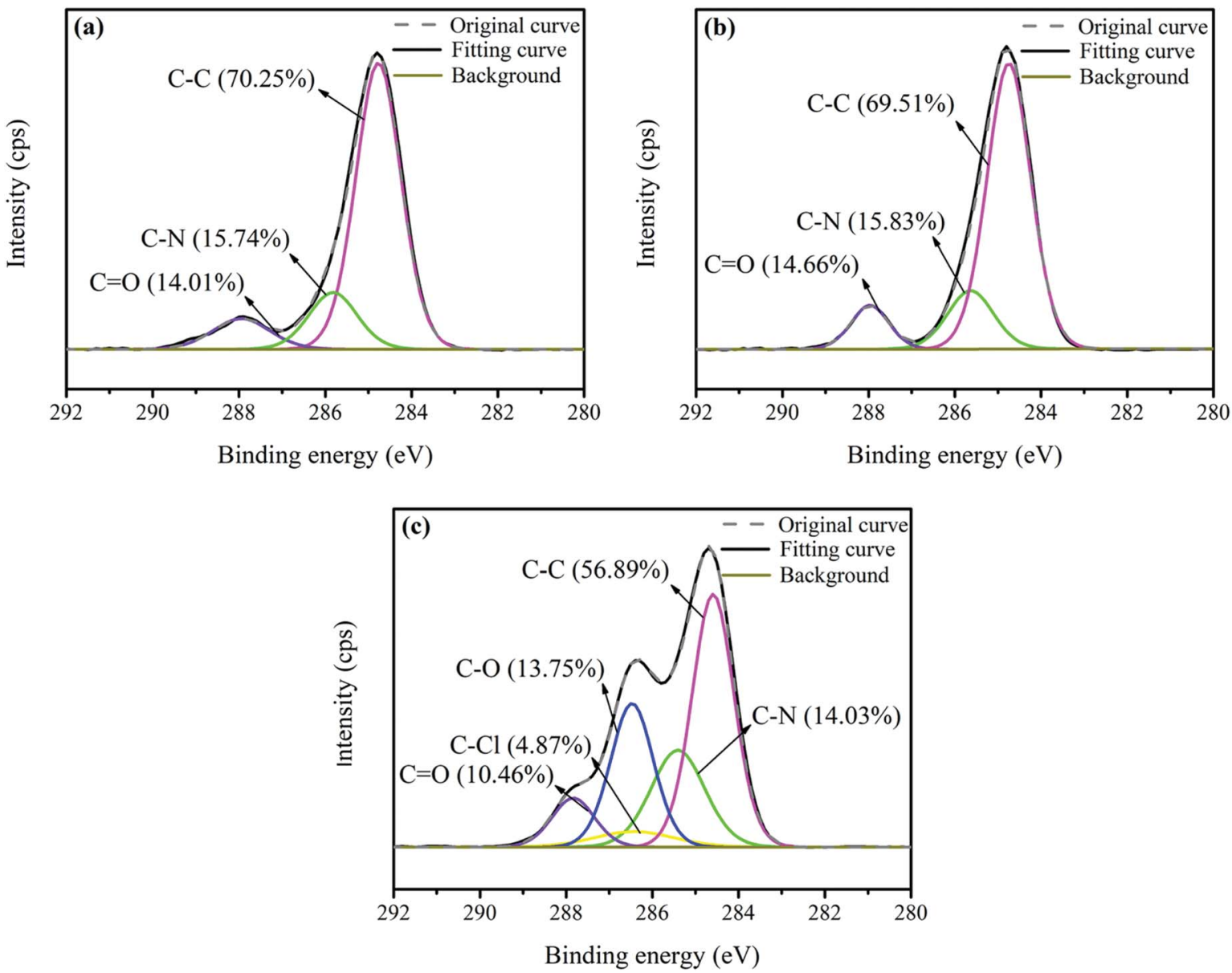

Fig. 6 The $\mathrm{C}_{1 \mathrm{~s}}$ peak spectra of the surfaces of (a) the untreated AFs, (b) AFs treated in pure $\mathrm{ScCO}_{2}$ and (c) AFs modified with $\mathrm{ECH}$ in $\mathrm{ScCO}$.

essential to emphasize that the presence of $\mathrm{ScCO}_{2}$ made the fiber surface smoother, which was verified from Fig. $7 \mathrm{~b}$. This was mainly due to the fact that $\mathrm{ScCO}_{2}$ could further extract the impurities from the fiber surface. ${ }^{14}$ An obvious morphology variation in the fiber surface was observed after treatment with ECH in $\mathrm{ScCO}_{2}$, as depicted in Fig. 7c. Specifically, some mild grooves and gibbous particles existed on the modified fiber surface, which had the advantage of creating a mechanical bite between the fiber and resin system to enhance the bond property between them.

\subsection{DCA analysis}

DCA is an important parameter to describe the wetting ability of the related fiber surfaces. Fig. $8 \mathrm{a}$ and $\mathrm{b}$ show the CA and surface free energy of the untreated and modified AF surfaces, respectively. No obvious changes in CA and surface free energy were found when the fibers were treated without $\mathrm{ECH}$ in $\mathrm{ScCO}_{2}$, which corresponded to the XPS and SEM results. CA dramatically decreased from $76.46^{\circ}$ to $46.47^{\circ}$ for water and from $37.46^{\circ}$ to $26.24^{\circ}$ for diiodomethane after modification with ECH in
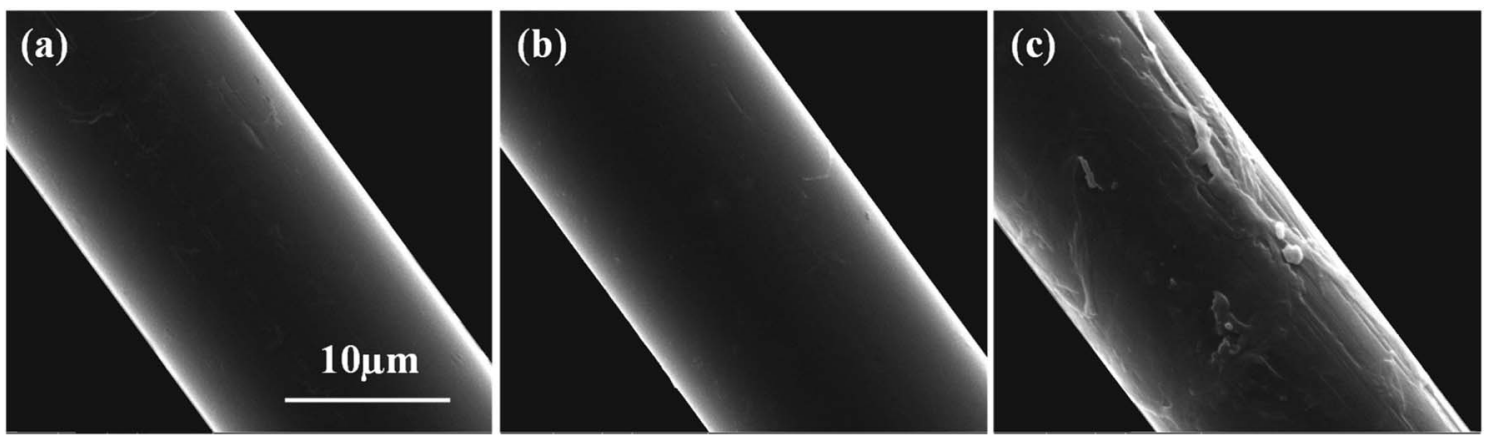

Fig. 7 The surface morphologies of (a) the untreated AFs, (b) AFs treated in pure $\mathrm{ScCO}_{2}$ and (c) AFs modified with $\mathrm{ECH}$ in $\mathrm{ScCO}_{2}$. 

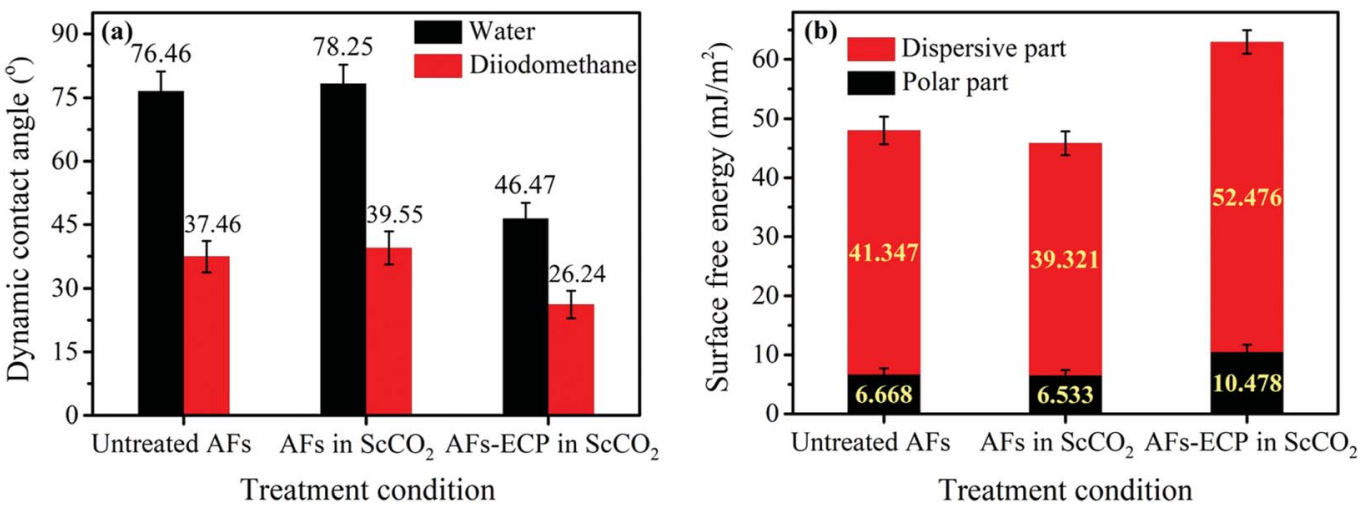

Fig. 8 (a) The CA and (b) surface free energy of the untreated and modified AF surfaces.

$\mathrm{ScCO}_{2}$. In addition, the polar and dispersive surface energies of the fiber surface increased to 10.48 and $52.48 \mathrm{~mJ} \mathrm{~m}^{-2}$, respectively, in comparison to those of the untreated materials: 6.67 and $41.35 \mathrm{~mJ} \mathrm{~m}^{-2}$, respectively. These results show the possibility of the improvement in the contact areas and wettability between AF and the resin system.

\subsection{IFSS property analysis}

During the service process of composite materials, stress is transferred between the matrix and reinforcement through their interfaces. Therefore, the bond strength between the interfaces directly affects the performance of composites. The single fiber micro-debonding test is often used to evaluate the IFSS property between the fiber and resin system. The IFSS properties of the untreated and modified AFs with the resin system are shown in Fig. 9a. Compared with the IFSS property of the untreated AFs, the IFSS property of the fibers treated with $\mathrm{ECH}$ in $\mathrm{ScCO}_{2}$ improved by $45.84 \%$, indicating an increase in the bond property between the fiber and the resin system. This was due to the rougher fiber surface and improvement in the oxygen-containing functional groups on the fiber surface. It is conceivable that the IFSS property of the fibers modified only with $\mathrm{ScCO}_{2}$ was lower than that of the untreated fibers, which was consistent with previous characterizations of the fiber surface. In addition, in order to verify the uniformity of the modification effect of the fiber surface modified with ECH in $\mathrm{ScCO}_{2}$, we randomly selected the fibers at three different positions in the reactor for the IFSS test, as shown in Fig. 1, and the IFSS properties are shown in Fig. 9b. We could conclude that the IFSS values of the modified fibers at three positions, namely, the upper, middle and lower portions of the reactor were very similar, indicating that the modification effect of the fiber surface modified with $\mathrm{ECH}$ in $\mathrm{ScCO}_{2}$ was very uniform.

\subsection{ILSS property analysis}

Since the poor adhesion between the fiber and the resin system is often the main cause of damage in composites, the ILSS property is usually used to characterize the degree of adhesion between the composite layers to evaluate the interlaminar property of the composites. Fig. 10a shows the ILSS property of the AF-reinforced composites and Fig. 10b presents the corresponding typical load-deflection of the composites. Compared with the ILSS property of the untreated AF-reinforced composites, the ILSS property of the AF-ECH-reinforced composites improved by $68.01 \%$, which indicated an increase in the bond property between the fiber and the resin system. The increase in
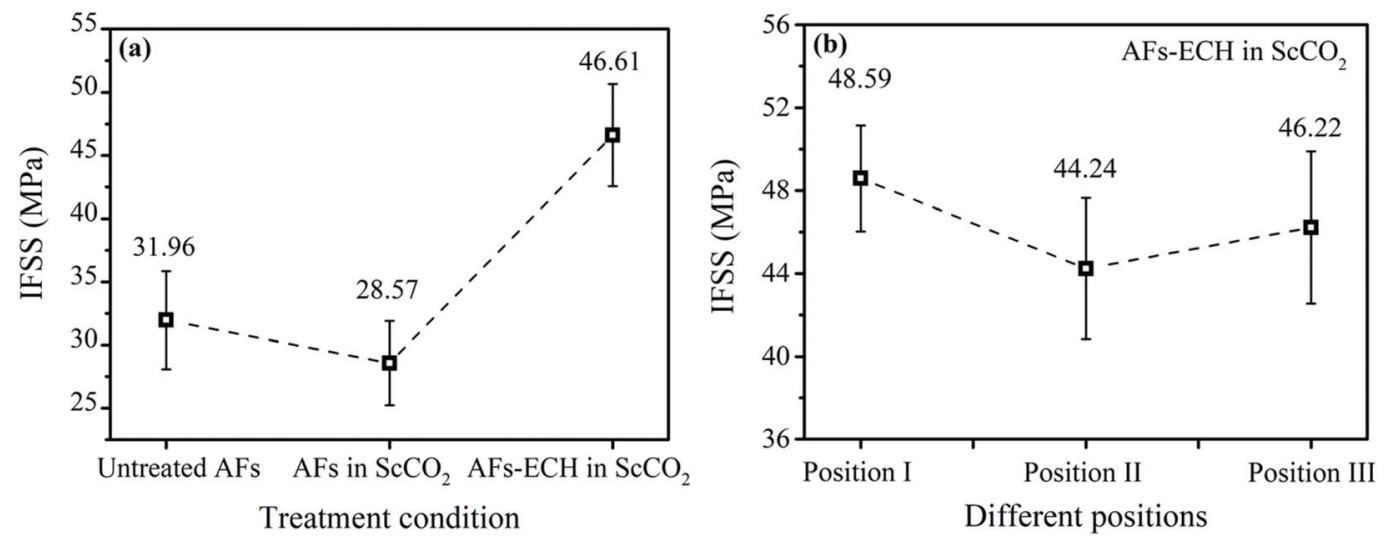

Fig. 9 The IFSS property of the untreated and modified AFs (a) and the IFSS property of AFs modified with $\mathrm{ECH}$ in $\mathrm{ScCO}_{2}$ at different positions in the reactor (b). 

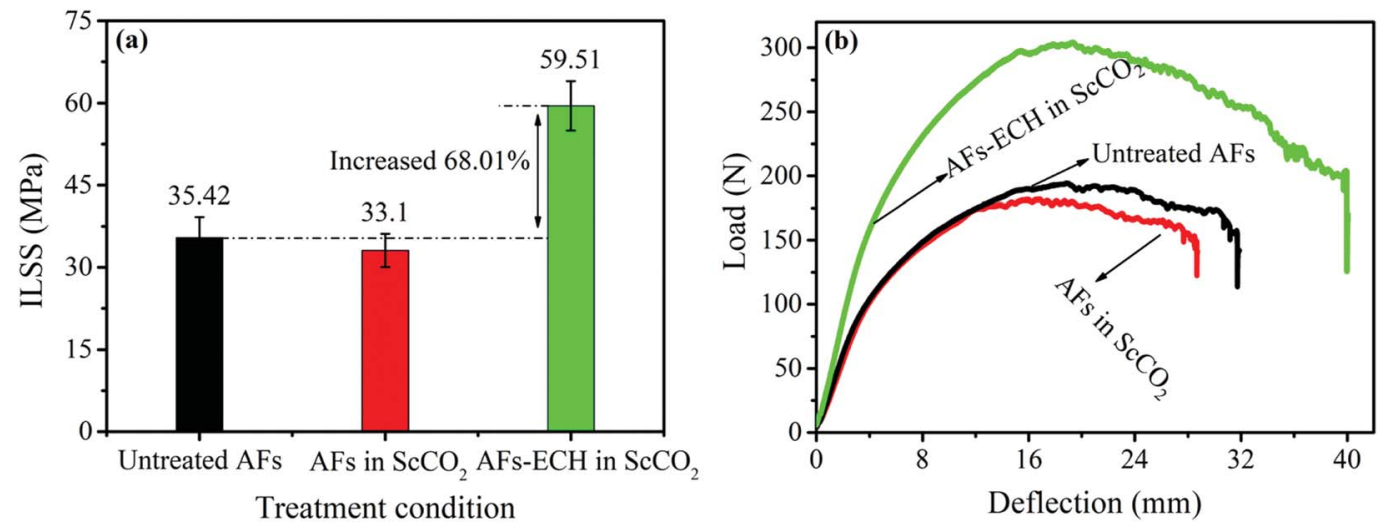

Fig. 10 The ILSS property (a) and the typical load-deflection (b) of the untreated and modified AF-reinforced resin composites.
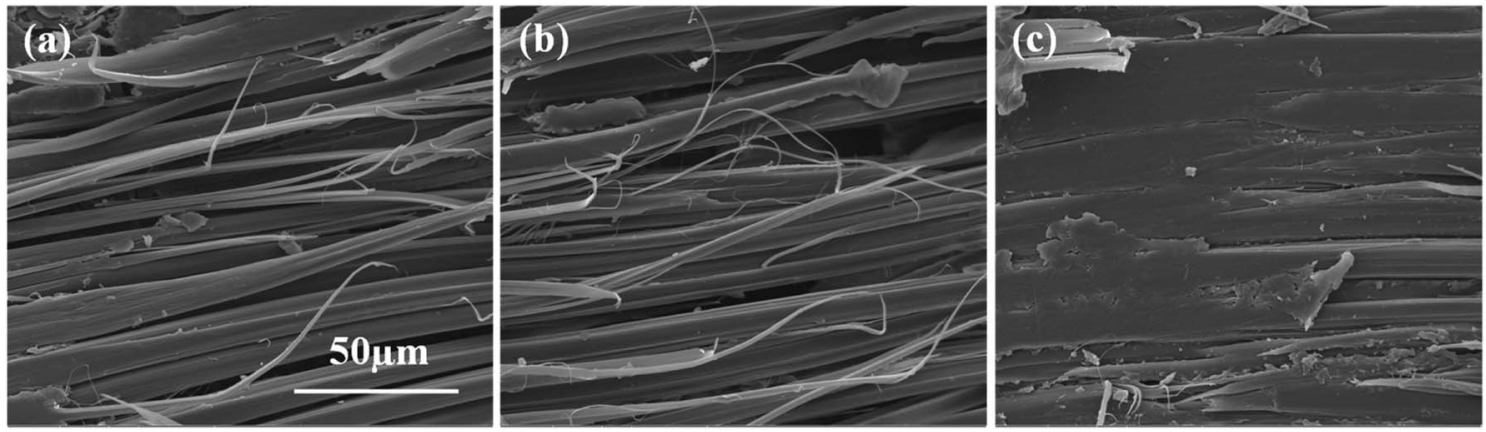

Fig. 11 The fracture cross-section surface of composites with AFs modified at different conditions: (a) the untreated AFs, (b) AFs treated in pure $\mathrm{ScCO}_{2}$ and (c) AFs modified with $\mathrm{ECH}$ in $\mathrm{ScCO}_{2}$.

the bond property can be explained from the following two aspects: first, the improvement in the relative oxygen content and the $\mathrm{O} / \mathrm{C}$ ratio indicated an increase in the oxygencontaining groups on the fiber surface, thereby increasing the activity of the fiber surface, which increased the bond property between the fiber and the resin system. Second, the surface roughness of AF-ECH increased, which added mechanical bites between the fiber and the resin system to some extent. It could be seen that $\mathrm{ScCO}_{2}$ was mainly present in the form of a kind of medium for carrying ECH to the fiber surface in the case of dynamic fluids, therefore forming an atmosphere full of ECH to modify the AF surface uniformly.

\subsection{SEM images of composite fracture surfaces}

Fig. 11 shows the SEM images of the cross-section fracture surface of the composites after interlaminar shear failure before and after the surface modification of fibers. It can be seen from Fig. 11a that there are almost no resins bonded on the fiber surface at the cross-section fracture surface of the untreated AFreinforced composites, indicating that the fiber and the resin system are separated during the fracture of the composite layer. This corresponded to the fact that the fiber surface was very smooth and not favorable for bonding with the resin. This fracture condition indicated that the fibers did not enhance the composite during the fracture process. A similar situation was also acquired when the fibers were treated in pure $\mathrm{ScCO}_{2}$, as shown in Fig. 11b. In contrast to this, a large amount of resin remained at the cross-section fracture surface during the destruction process of the composite layers when the fibers were modified with $\mathrm{ECH}$ in $\mathrm{ScCO}_{2}$, as shown in Fig. 11c, indicating that a good interface between the fiber and the resin system was formed. The fracture condition indicated that the fibers had a reinforcing effect on the composite materials and enhanced the ILSS performance of the composite materials.

\section{Conclusions}

This paper proposed a method for grafting $\mathrm{ECH}$ on the AF surface using the $\mathrm{AlCl}_{3}$ catalyst assisted by $\mathrm{ScCO}_{2}$ in a reactor. The surface modification made the fiber surface rougher and more active to form a better interface for combining with the resin system. In addition, an increase of $45.84 \%$ in the IFSS property and an increase of $68.01 \%$ in the ILSS property both powerfully illustrated that it was an effective way to improve the interface combination between $\mathrm{AF}$ and the resin system. Moreover, as a green and environmentally friendly medium, $\mathrm{ScCO}_{2}$ can simultaneously modify fiber products in a wide range.

\section{Conflicts of interest}

There are no conflicts to declare. 


\section{Acknowledgements}

This work was supported by the State Key Laboratory for Modification of Chemical Fibers and Polymer Materials, Donghua University (No. LK1602).

\section{References}

1 W. Tian, Q. Teng, Y. Shi, L. He and X. Tuo, Mater. Lett., 2017, 202, 158-161.

2 B. A. Patterson, M. H. Malakooti, J. Lin, A. Okorom and H. A. Sodano, Compos. Sci. Technol., 2018, 161, 92-99.

3 G. Qi, B. Zhang and S. Du, Composites, Part A, 2018, 121, 549557.

4 H.-D. Zheng, J. Zhang, J. Yan and L.-J. Zheng, RSC Adv., 2017, 7, 3470-3479.

5 S. Ifuku, H. Maeta, H. Izawa, M. Morimoto and H. Saimoto, RSC Adv., 2014, 4, 40377-40380.

6 C. Zheng, D. Hong, D. Yu, J. Chan, C. Meng, L. Luo and X. Liu, Appl. Surf. Sci., 2018, 434, 473-480.

7 H. Cen, Y. L. Kang, Z. K. Lei, Q. H. Qin and W. Qiu, Compos. Struct., 2006, 75, 532-538.

8 C. Zheng, H. Yutong, L. Longbo and L. Xiangyang, Mater. Lett., 2018, 233, 102-106.

9 B. A. Patterson and H. A. Sodano, ACS Appl. Mater. Interfaces, 2016, 8, 33963-33971.

10 Y. Dan, S. Mu, L. Liu and W. Wei, Colloids Surf., A, 2015, 483, 53-59.

11 J. R. Chen, Y. F. Zhu, Q. Q. Ni, Y. Q. Fu and X. Fu, Appl. Surf. Sci., 2014, 321, 103-108.

12 W. Fan, H. Tian, H. Wang, T. Zhang and S. Wang, Polym. Test., 2018, 72, 147-156.

13 S. Asai, Y. Shimada, Y. Tominaga and M. Sumita, Macromolecules, 2005, 38, 6544-6550.

14 A. Cooper, J. Mater. Chem., 2000, 10, 207-234.

15 H. Kong, C. Teng, X. Liu, J. Zhou and M. Yu, RSC Adv., 2014, 4, 20599-20604.

16 X. Wang, W. Cui, C. Meng and Q. Xu, Mater. Lett., 2017, 201, 129-132.

17 J. Yue, Q. Xu, Z. W. Zhang and Z. M. Chen, Macromolecules, 2007, 40, 8821-8826.

18 X. Zhao, K. Hirogaki, I. Tabata, S. Okubayashi and T. Hori, Surf. Coat. Technol., 2006, 201, 628-636.
19 M. Belmas, I. Tabata, K. Hisada and T. Hori, J. Appl. Polym. Sci., 2011, 119, 2283-2291.

20 N. Martinez, K. Hisada, I. Tabata, K. Hirogaki, S. Yonezawa and T. Hori, J. Supercrit. Fluids, 2011, 56, 322-329.

21 H. J. Kong, P. Yang, C. Q. Teng and M. H. Yu, RSC Adv., 2015, 5, 58916-58920.

22 X. Jing, Y. Han, L. Zheng and H. Zheng, J. CO2 Util., 2018, 27, 289-296.

23 H. Kong, Q. Xu and M. Yu, Polymers, 2019, 11, 1110-1123.

24 H. Yoshida, M. Sone, H. Wakabayashi, H. Yan, K. Abe, X. T. Tao, A. Mizushima, S. Ichihara and S. Miyata, Thin Solid Films, 2004, 446, 194-199.

25 J. Wu and X. H. Cheng, J. Appl. Polym. Sci., 2006, 102, 41654170.

26 T. M. Liu, Y. S. Zheng and J. Hu, J. Appl. Polym. Sci., 2010, 118, 2541-2552.

27 Y. C. Ling, H. C. Teng and C. Cartwright, J. Chromatogr. A, 1999, 835, 145-157.

28 B. Li, W. Guo, W. Song and E. D. Ramsey, J. Chem. Eng. Data, 2016, 61, 2128-2134.

29 M. Álvaro, D. Das, M. Cano and H. Garcia, J. Catal., 2003, 219, 464-468.

30 Y. Gu, X. Tan, Z. Yang, L. Min and Z. Zhang, Mater. Des., 2014, 56, 852-861.

31 Z. Guo, D. Ding, X. Yan, X. Zhang, Y. D. Huang, S. Wei, L. Xing, Q. Zhang, Z. Wu and J. Guo, J. Mater. Chem. A, 2014, 2, 18293-18303.

32 L. Chen, Y. Du, Y. Huang, P. F. Ng and B. Fei, Compos. Sci. Technol., 2016, 129, 86-92.

33 W. W. Zhou, G. Yamamoto, Y. Fan, H. Kwon, T. Hashida and A. Kawasaki, Carbon, 2016, 106, 37-47.

34 Z. Jiang, A. Imam, R. Crane, K. Lozano, V. N. Khabashesku and E. V. Barrera, Compos. Sci. Technol., 2007, 67, 1509-1517.

35 Z. Cheng, P. Wu, B. Y. Li, T. Chen, Y. Liu, M. M. Ren, Z. M. Wang, W. C. Lai, X. Wang and X. Y. Liu, Appl. Surf. Sci., 2016, 384, 480-486.

36 Z. Shu-Hui, H. E. Guo-Qiang, L. Guo-Zheng, H. Cui, W. Zhang and B. Wang, Appl. Surf. Sci., 2010, 256, 21042109.

37 Y. H. Zhang, Y. D. Huang, L. Liu and K. L. Cai, Appl. Surf. Sci., 2008, 254, 3153-3161.

38 W. Jing, C. Ping, C. Lu, Y. Qi, L. Wei and R. Rong, Compos. Interfaces, 2018, 25, 1-13. 